\title{
Objective Measurement of Cosmetic Outcomes of Breast Conserving Therapy Using BCCT.core
}

\section{Tosol Yu, MD' \\ Keun-Yong Eom, MD, PhD' \\ Na Young Jang, MD, $P h D^{1}$ \\ Kyung Su Kim, MD 1 \\ Tae Ryool Koo, MD' \\ Jeanny Kwon, MD \\ Byoung Hyuck Kim, MD ${ }^{1}$ \\ Eunyoung Kang, $\mathrm{MD}, \mathrm{PhD}^{2}$ \\ Sung-Won Kim, MD, PhD² \\ Jae-Sung Kim, MD, PhD' \\ In Ah Kim, MD, PhD}

Departments of ${ }^{1}$ Radiation Oncology and ${ }^{2}$ Surgery, Seoul National University

Bundang Hospital, Seongnam, Korea
Correspondence: In Ah Kim, MD, PhD

Department of Radiation Oncology,

Seoul National University Bundang Hospital,

82 Gumi-ro 173Beon-gil, Bundang-gu,

Seongnam 13620, Korea

Tel: $82-31-787-7651$

Fax: 82-31-787-4019

E-mail: inah228@snu.ac.kr

Received March 2, 2015

Accepted May 11, 2015

Published Online June 22, 2015

\section{Purpose}

The purpose of this study is to evaluate objective cosmetic outcomes and factors related to breast-conserving therapy (BCT) using the BCCT.core software.

\section{Materials and Methods}

Fifty-one patients who received BCT with informed consent were evaluated using the BCCT.core software. Patients were divided into two groups based on the BCCT score: excellent or good ( $n=42)$ vs. fair or poor $(n=9)$. Analysis of clinical factors was performed to determine factors affecting cosmetic outcomes.

\section{Results}

The objective cosmetic outcome of BCT measured using the BCCT.core software was excellent in $10 \%$ of patients, good in $72 \%$, and fair in $18 \%$. None of the patients were classified as poor outcome. Tumor characteristics, systemic adjuvant therapy (chemotherapy and hormonal therapy), and radiation dose or energy of electron boost did not show correlation with the score measured by the BCCT.core program $(p>0.05)$. In univariate analysis, maximum dose within the breast $\left(D_{\max }\right)$, width of tangential field, and excised tumor volume were smaller in patients with excellent or good by the BCCT.core compared to those with fair or poor $\left(D_{\max }, 110.2 \pm 1.5 \%\right.$ vs. $111.6 \pm 1.7 \%, p=0.019$; width of tangential field, $8.0 \pm 1.1$ $\mathrm{cm}$ vs. $8.6 \pm 0.7 \mathrm{~cm}, \mathrm{p}=0.034$; excised tumor volume, $64.0 \pm 35.8 \mathrm{~cm}^{3}$ vs. $95.3 \pm 54.4 \mathrm{~cm}^{3}$, $\mathrm{p}=0.067$ ). In multivariate analysis, only $D_{\max }$ was a significant factor for breast cosmetic outcome with a risk ratio of 1.697 (95\% confidence interval, 1.006 to $2.863 ; p=0.047$ ).

\section{Conclusion}

Objective measurement of cosmetic outcome of BCT using the BCCT.core software was feasible. The cosmetic outcome of BCT may be affected by the maximum dose within the breast.

\author{
Key words \\ Breast neoplasms, Breast conservative therapy, \\ Quality of life, BCCT.core, Esthetics
}

\section{Introduction}

Breast conserving therapy (BCT) has been a preferred treatment method for early breast cancer since equivalent loco-regional control and overall survival with improved cosmetic outcome compared with mastectomy was reported in several studies [1]. The Harvard scale proposed by Harris et al. [2], which has been used to evaluate cosmetic outcome after BCT, is a 4-point scale that describes the global esthetic appearance of breasts as excellent, good, fair, or poor. This scale is simple and not time-consuming; however, its interobserver concurrence is poor and the outcome can be affected by the experience level of the observers [3]. 
To overcome this subjectivity in breast cosmesis evaluation, a more objective scale such as breast retraction assessment (BRA) can be used alternatively [4]. The BRA evaluates the distances from the sternal notch to nipples and from the nipples to breast outline. Because the BRA uses only quantitative values, the concurrence between observers is excellent. However, this scale does not include other factors that might be responsible for a poor cosmetic result such as skin alteration or disturbing scars [5].

For use in the clinic, an evaluation tool should be both simple and objective. The BCCT.core program might be an appropriate tool that meets these conditions, and its feasibility was previously reported [6,7]. The BCCT.core software developed by the Breast Research Group (INSEC Porto, the University of Porto) evaluates cosmetic outcomes automatically using photographs of a patient. The program categorizes cosmetic outcome as one of four categories (excellent, good, fair, and poor) based on asymmetry score, color difference, and surgical scar [8]. Cosmetic evaluation by BCCT.core software is not highly dependent on the observer and several studies reported a weighted kappa score for inter-observer agreement of 0.87 to 0.88 [9,10]. The correlation between the Harvard scale and BCCT.core is reported as moderate to fair $(\mathrm{K}=0.21-0.57)$, and the inter-observer variation is less than that for the Harvard scale $[7,9,10]$.

In this study, cosmetic outcomes of patients treated with BCT were evaluated using BCCT.core software and predictive factors were analyzed.

\section{Materials and Methods}

Patients who underwent BCT at our hospital with informed consent from 2005 to 2011 were eligible for this study. This work was approved by the institutional review board. Photographs of 51 enrolled patients were taken at the outpatient clinic during the follow-up. The median interval between the end of whole breast radiotherapy and photographic evaluation was 26 months (range, 16 to 73 months). After the rating system was explained in detail, patients were asked to rate their body image changes using four options, excellent, good, fair, and poor [2]. The Harvard 4-point scale was used as the patient self-assessment rating method. The patient-reported cosmetic outcome was assessed with the cooperation of a researcher not involved in either the treatment or analysis of this study. Blue dot marks were placed $25 \mathrm{~cm}$ inferior from the sternal notch as a reference scale. Patients stood at attention on the fixed footprints and medical photos were taken using a Nikon D700 (Nikon, Tokyo, Japan) with a $60 \mathrm{~mm}$ lens. Only the front view was pho- tographed and the same position and lighting were used for all patients in order to minimize any possible skin color differences caused by the angle or intensity of the lighting. Two light sources were used for each photo, placed $2 \mathrm{~m}$ from the patient at a $45^{\circ}$ angle, left and right. The camera shutter speed was set a 1/125 and aperture at f14. Each photo was assessed using BCCT.core.

When loading a photograph on the BCCT.core software, users should adjust the red dots to the most medial and lateral point of the breast outline (Fig. 1). After pressing the "Auto Adjust" button, the breast outline and overall cosmetic result will be automatically calculated. In detail, the cosmetic results consist of three categories; asymmetry, color, and scar. The program uses seven variables for the asymmetry index: BRA, lower breast contour (LBC), upward nipple retraction, breast compliance evaluation, breast contour difference (BCD), breast area difference, and breast overlap difference (BOD), which measures the non-overlapping area of the two breasts [8]. Definitions of the variables for the asymmetry index are shown in Supplementary Table 1 and Fig. 1C. The 3D color histogram, consisting of 512 bins, was computed for measurement of the dissimilarity between two breasts. The differences in color and scar were then computed using the chi-square statistics and the Earth Mover's distance, a measure of the distance between two probability distributions over a region. The scores of asymmetry, color, and scar are then integrated into the overall rates.

All patients received 50-50.4 Gy of whole breast radiotherapy (WBRT) in 25 to 28 fractions with or without electron boost (9-16 Gy in 5 to 8 fractions) by a linear accelerator. WBRT was planned as a tangential field with an appropriate wedge using a computed tomography-based radiotherapy treatment planning system. The width of the tangential field was determined to be $1.5-2.0 \mathrm{~cm}$ larger than the depth of the breast. Excised tissue volume was estimated using the formula for calculating the volume of an ellipsoid body:

$$
\mathrm{V}=\frac{4}{3} \pi \times \mathrm{x} \times \mathrm{y} \times \mathrm{z}
$$

$\mathrm{D}_{\max }$ indicates the maximum point dose within the breast.

Agreement between the patients' self-rating score and the results of BCCT.core was determined by the kappa statistic. Statistical analyses were performed to determine factors affecting cosmetic outcomes using the independent $t$ test for continuous variables with normal distribution, Mann-Whitney $U$ test for continuous variables without normal distribution, and chi-square test for categorical variables. The variables were also assessed using a logistic regression model with a forward conditional method. The score of BCCT.core was dichotomized according to excellent or good and fair or poor, and a binary variable was used in those statistical analyses. 

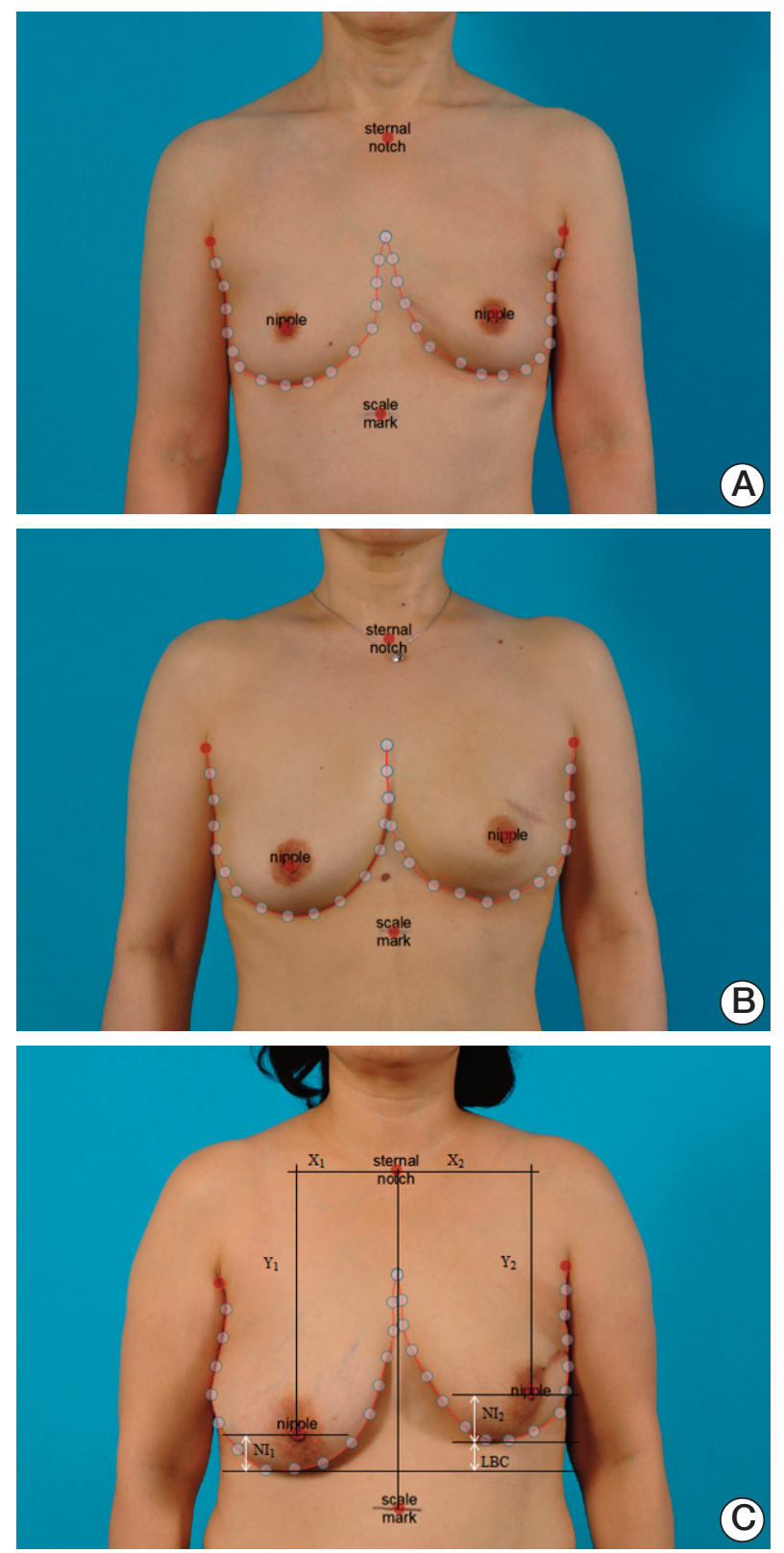

Fig. 1. Cosmetic results by BCCT.core. Representative examples of excellent outcome (A), good outcome (B), and fair outcome (C) are shown.

\section{Results}

Patients' characteristics are summarized in Table 1. The median age of the 51 patients was 54 years (range, 40 to 80 years). The majority of patients $(76 \%)$ had T1 stage disease, and 15 patients $(29 \%)$ had axillary lymph node metastases. Primary tumors were located in the upper outer quadrant in 29 patients (57\%).
The cosmetic outcome of BCT measured using BCCT.core software was rated as excellent in $10 \%$ of patients, good in $72 \%$, and fair in $18 \%$. None of the patients were rated as poor outcome. The details of asymmetry scores are summarized in Supplementary Table 1. The kappa value between scores by BCCT.core and by patient self-assessment was 0.12 , indicating slight agreement. Among the breast asymmetry indices, the score for BRA, $\mathrm{BBC}, \mathrm{BCD}$, and BOD showed significant correlation with the overall score (Table 2). In 
Table 1. Patient characteristics

\begin{tabular}{|c|c|}
\hline Characteristic & No. $(\%)$ \\
\hline \multicolumn{2}{|l|}{ T stage } \\
\hline Tis & $5(10)$ \\
\hline T1 & $39(76)$ \\
\hline $\mathrm{T} 2$ & $6(12)$ \\
\hline T3 & $1(2)$ \\
\hline \multicolumn{2}{|l|}{ N stage } \\
\hline No & $32(63)$ \\
\hline $\mathrm{N}+$ & $15(29)$ \\
\hline $\mathrm{Nx}$ & $4(8)$ \\
\hline \multicolumn{2}{|l|}{ Location $^{\text {a) }}$} \\
\hline UOQ & $29(57)$ \\
\hline UIQ & $12(24)$ \\
\hline LOQ & $4(8)$ \\
\hline LIQ & $2(4)$ \\
\hline Central & $3(6)$ \\
\hline \multicolumn{2}{|l|}{ Surgery } \\
\hline WE only & $4(8)$ \\
\hline WE+SLNBx & $30(59)$ \\
\hline WE+ALND & $14(27)$ \\
\hline WE with nipple excision & $3(6)$ \\
\hline \multicolumn{2}{|l|}{ Radiotherapy dose } \\
\hline WBRT 50.4 Gy & $8(16)$ \\
\hline WBRT 50.4 Gy+boost 9-10 Gy & $39(76)$ \\
\hline WBRT 50.4 Gy+boost 14.4-16 Gy & $4(8)$ \\
\hline \multicolumn{2}{|l|}{ Radiotherapy technique } \\
\hline Tangential field & $41(80)$ \\
\hline Tangential field with supraclavicular field & $10(20)$ \\
\hline \multicolumn{2}{|l|}{ Chemotherapy } \\
\hline None & $22(43)$ \\
\hline $\mathrm{AC} \pm \mathrm{D}$ & $18(35)$ \\
\hline $\mathrm{CMF}$ & $7(14)$ \\
\hline Others & $4(8)$ \\
\hline Total & $51(100)$ \\
\hline
\end{tabular}

UOQ, upper outer quadrant; UIQ, upper inner quadrant; LOQ, lower outer quadrant; LIQ, lower inner quadrant; WE, wide excision; SLNBx, sentinel lymph node biopsy; ALND, axillary lymph node biopsy; WBRT, whole breast radiotherapy; $\mathrm{AC}$, cyclophosphamide and doxorubicin; $\mathrm{D}$, docetaxel; CMF, cyclophosphamide, methotrexate, and 5-fluorouracil. a)Available data only.

particular, the Pearson correlation score of LBC, which indicates the difference between the levels of the inferior breast contours, was the highest (Pearson's coefficient $=0.552$, $p<0.001$ ). However, none of the indices showed correlation with the patients' self-assessment.

Patients classified in the good to excellent group had a lower maximum point dose within the breast than patients
Table 2. Correlations between breast asymmetry indices and overall cosmetic outcomes of BCCT.core and between breast asymmetry indices and patients' self-assessment scores

\begin{tabular}{lccrrc} 
& \multicolumn{2}{c}{ BCCT.core } & & \multicolumn{2}{c}{ Patients' self-assessment } \\
\cline { 2 - 3 } \cline { 5 - 6 } & Pearson & p-value & & Pearson & p-value \\
BRA & 0.278 & 0.048 & & 0.233 & 0.111 \\
LBC & 0.552 & $<0.001$ & & -0.084 & 0.568 \\
UNR & 0.219 & 0.122 & & 0.169 & 0.250 \\
BCE & 0.204 & 0.151 & & 0.068 & 0.645 \\
BCD & 0.390 & 0.005 & & -0.082 & 0.580 \\
BAD & 0.245 & 0.084 & & 0.065 & 0.663 \\
BOD & 0.438 & 0.001 & & -0.056 & 0.705 \\
\hline
\end{tabular}

BRA, breast retraction assessment; LBC, lower breast contour; UNR, upward nipple retraction; $\mathrm{BCE}$, breast compliance evaluation; $\mathrm{BCD}$, breast contour difference; $\mathrm{BAD}$, breast area difference; $\mathrm{BOD}$, breast overlap difference.

in the fair to bad group $(110.2 \pm 1.5 \%$ vs. $111.6 \pm 1.7 \%, \mathrm{p}=0.019)$ (Table 3). The width of the tangential field was smaller in patients who had a good or excellent outcome than in patients with fair or bad results $(8.0 \pm 1.1 \mathrm{~cm}$ vs. $8.6 \pm 0.7 \mathrm{~cm})$, but the length of the tangential field did not show significant correlation with the cosmetic outcome.

Regarding surgical factors, large excised tissue volume showed a trend toward worse cosmetic outcome $(64.0 \pm 35.8$ $\mathrm{cm}^{3}$ vs. $\left.95.3 \pm 54.4 \mathrm{~cm}^{3}, \mathrm{p}=0.067\right)$, but excised skin area and tumor volume were not significant factors for cosmetic outcome.

Other factors including age, histologic type and other histologic findings, tumor location, re-excision, menopause, nipple excision, axillary lymph node dissection, chemotherapy regimen and other adjuvant therapies, and electron boost did not show significant correlation with cosmetic outcomes (Table 3).

Binary logistic regression analysis was performed using maximum point dose within breast, width of tangential field, and excised tissue volume as variables. Only $\mathrm{D}_{\max }$ was a significant factor for breast cosmetic outcome with a risk ratio of 1.697 (Table 4).

\section{Discussion}

With early detection of breast cancer and an increase in the cure rate, the cosmetic aspect of breast cancer treatment is becoming more important. The shift in the treatment para- 
Table 3. Univariate analysis for factors affecting the cosmetic outcome

\begin{tabular}{|c|c|c|c|c|}
\hline \multirow{2}{*}{ Factor } & \multirow{2}{*}{ No. of patients (\%) } & \multicolumn{2}{|c|}{ Cosmetic outcome by BCСТ.core } & \multirow{2}{*}{ p-value } \\
\hline & & Excellent or good $(\%)$ & Fair or bad $(\%)$ & \\
\hline Age (yr) & & $54.8 \pm 9.3$ & $57.5 \pm 6.9$ & 0.601 \\
\hline Menopause & $31(61)$ & $24(47)$ & $5(10)$ & 0.723 \\
\hline \multicolumn{5}{|l|}{ Histology } \\
\hline DCIS & $9(18)$ & $7(14)$ & $2(4)$ & 0.589 \\
\hline LCIS & $1(2)$ & $1(2)$ & 0 & \\
\hline IDC & $39(76)$ & $33(65)$ & $6(12)$ & \\
\hline ILC & $2(4)$ & $1(2)$ & $1(2)$ & \\
\hline \multicolumn{5}{|l|}{ Location of tumora) } \\
\hline UOQ & $29(57)$ & $24(47)$ & $5(10)$ & 0.763 \\
\hline UIQ & $12(24)$ & $11(22)$ & $1(2)$ & \\
\hline LOQ & $4(8)$ & $3(7)$ & $1(2)$ & \\
\hline LIQ & $2(4)$ & $2(4)$ & 0 & \\
\hline Central & $3(6)$ & $2(4)$ & $1(2)$ & \\
\hline Longest diameter of tumor $(\mathrm{cm})$ & & $1.6 \pm 0.8$ & $2.0 \pm 1.5$ & 0.635 \\
\hline Tumor volume $\left(\mathrm{cm}^{3}\right)$ & & $3.4 \pm 4.4$ & $8.8 \pm 18.6$ & 0.561 \\
\hline Re-excision rate & $46(90)$ & $38(75)$ & $8(16)$ & 0.884 \\
\hline Nipple excision rate & $3(6)$ & $2(4)$ & $1(2)$ & \\
\hline Axillary lymph node dissection rate & $14(27)$ & $11(22)$ & $3(6)$ & 0.663 \\
\hline Excised tissue volume $\left(\mathrm{cm}^{3}\right)$ & & $64.0 \pm 35.8$ & $95.3 \pm 54.4$ & 0.067 \\
\hline Excised skin area $\left(\mathrm{cm}^{2}\right)$ & & $6.1 \pm 3.1$ & $14.1 \pm 9.8$ & 0.294 \\
\hline \multicolumn{5}{|l|}{ Chemotherapy regimen } \\
\hline Not done & $22(43)$ & $18(35)$ & $4(8)$ & 0.217 \\
\hline $\mathrm{AC} \pm \mathrm{D}$ & $18(35)$ & $16(31)$ & $2(4)$ & \\
\hline $\mathrm{CMF}$ & $7(14)$ & $4(8)$ & $3(6)$ & \\
\hline Others & $4(8)$ & $4(8)$ & 0 & \\
\hline Herceptin rate & $3(6)$ & $2(4)$ & $1(2)$ & 0.463 \\
\hline Hormonal therapy rate & $11(22)$ & $9(18)$ & $2(4)$ & 0.958 \\
\hline Length of tangential field (cm) & & $18.1 \pm 1.7$ & $18.4 \pm 1.6$ & 0.790 \\
\hline Width of tangential field (cm) & & $8.0 \pm 1.1$ & $8.6 \pm 0.7$ & 0.034 \\
\hline $\mathrm{D}_{\max }(\%)$ & & $110.2 \pm 1.5$ & $111.6 \pm 1.7$ & 0.019 \\
\hline \multicolumn{5}{|l|}{ Electron boost } \\
\hline Not done & $8(16)$ & $7(14)$ & $1(2)$ & 0.542 \\
\hline 9-10 Gy & $39(76)$ & $31(61)$ & $8(16)$ & \\
\hline 14.4-16 Gy & $4(8)$ & $4(8)$ & 0 & \\
\hline Supraclavicular field rate & $41(80)$ & $33(65)$ & $8(16)$ & 0.479 \\
\hline
\end{tabular}

DCIS, ductal carcinoma in situ; LCIS, lobular carcinoma in situ; IDC, infiltrative ductal carcinoma; ILC, infiltrative lobular carcinoma; UOQ, upper outer quadrant; UIQ, upper inner quadrant; LOQ, lower outer quadrant; LIQ, lower inner quadrant; AC, cyclophosphamide and doxorubicin; D, docetaxel; CMF, cyclophosphamide, methotrexate, and 5-fluorouracil; $\mathrm{D}_{\text {max, }}$

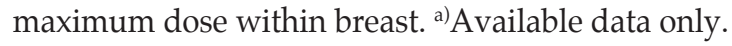

digm from mastectomy to breast conserving treatment is overwhelming, therefore, many studies now use cosmetic outcome as a secondary endpoint. Several factors affecting cosmetic outcome have been reported; however, most studies used subjective evaluation methods and few studies evaluating cosmetic outcome using objective tools such as BCCT.core software have been reported.
In our study, when using BCCT.core as a method for evaluating the cosmetic outcome, the maximum point dose within the breast was the only significant factor in multivariate analysis. The side effects of radiotherapy on breast tissue include skin changes and responses of subcutaneous tissue and parenchyma. The skin change usually resolves in 1-3 months; however, hypo- or hyper-pigmentation or fibro- 
Table 4. Multivariate analysis for factors affecting the cosmetic outcome

\begin{tabular}{lccc} 
Factor & Hazard ratio & $95 \% \mathrm{CI}$ & p-value \\
Excised tissue volume $\left(\mathrm{cm}^{3}\right)$ & 1.008 & $0.999-1.017$ & 0.082 \\
Width of tangential field $(\mathrm{cm})$ & 0.959 & $0.389-2.367$ & 0.928 \\
$\mathrm{D}_{\max }(\%)$ & 1.697 & $1.006-2.863$ & 0.047 \\
\hline
\end{tabular}

CI, confidential interval; $D_{\max }$ maximum dose within breast.

sis may persist for a longer period [11]. In this regard, BCCT.core reflects not only breast symmetry but also objective measurement of pigmentation and scar, which are also important factors in cosmetic outcome. Several radiotherapyrelated factors including dose homogeneity were reported to affect cosmetic outcomes and dosimetric variables such as $\mathrm{D}_{\max }(\%)$ and percent volume of breast tissue receiving the given dose have been used to evaluate dose homogeneity. Hepel et al. [12], who evaluated the influence of dosimetric variables on cosmetic outcomes of patients treated with accelerated partial breast irradiation, reported that the percent volume of breast tissue receiving $5 \%$ and $20 \%$ of the prescription dose and maximum point dose showed correlation with the risk of fibrosis.

In this study, in univariate analysis, the width of the tangential field was also a factor affecting cosmesis. Dose inhomogeneity is usually related to a larger breast, and the width of the field reflects the size of the breast, however it is a relatively imprecise metric $[13,14]$. Intensity modulated radiotherapy (IMRT), instead of the conventional 2D or 3D technique, is performed in several institutes to improve dose homogeneity and reduce the high-dose area in the treated breast. Harsolia et al. [15], who compared late toxicities between conventional whole breast irradiation and breast IMRT, reported reduced hyperpigmentation and edema in the IMRT arm. These trends were more significant in patients with larger breasts. Better cosmetic outcome might be achieved by efforts to minimize the undesirable high-dose region within the breast [16].

Use of boost in WBRT is also an important issue. Randomized trials have shown that a boost to the surgical bed improves local control; however, several studies reported worse cosmetic outcome with boost radiotherapy [1,17]. Among patients who received a 25-Gy boost in the EORTC 10801 trial, $30 \%$ had a fair or poor cosmetic result at the 3-year follow up. The EORTC 22881/10882 trial also reported significantly poor cosmetic results in patients who received a boost of $16 \mathrm{~Gy}$. In contrast, in the Lyon trial no difference in self-assessed cosmesis was observed between the boost and no boost group, although the fractionation of WBRT was not conventional [18]. Taylor et al. [19] reported that use of boost was not a significant factor of cosmetic outcome, while the whole breast dose was a significant factor. Several other studies also reported that whole breast dose is more important than boost radiotherapy with respect to cosmetic outcome [20]. However, all of the above mentioned studies used a subjective 4-point scale as a cosmesis evaluation tool, therefore an objective tool such as the BCCT.core should be used for more accurate comparison in future studies.

In this study, although no patient- or tumor-related factors showed correlation with cosmetic outcome, correlation of some factors with cosmesis was reported in other studies. Older age was reported as a worse predictive factor for cosmetic outcome in several studies $[4,19,21]$. In a study from Washington University, cosmetic outcome scored by either the patient or physician was worse in patients with age over 60 years $(p=0.001)$, postmenopausal status $(p=0.02)$, and black race $(\mathrm{p}=0.0034)$. Tumor size did not show correlation with breast cosmesis in our study; however, it was reported as an important factor in several other studies $[19,21]$.

Several surgical factors were also reported as significant factors in previous studies. Pezner et al. [4], who evaluated cosmetic outcome of patients who received BCT using the BRA method, reported that patients who underwent extensive tumor resection had greater retraction than those who underwent a more limited resection. Data from other institutes in Korea also identified the extent of tumor resection as a significant factor in cosmetic outcome evaluated by the physician using the subjective Harvard scale [22], and other studies reported similar results $[19,23]$. In our study the volume of resected tumor or post-surgical defects, which can affect breast asymmetry after $\mathrm{BCT}$, showed a marginal association with the cosmetic outcome by BCCT.core. Most patients had a $\mathrm{T} 1$ tumor $(76 \%)$; the primary tumor resection was performed with relative uniformity by one surgeon, with wide excision and a median resected volume of $61 \mathrm{~cm}^{3}$. The high proportion of $\mathrm{T} 1$ tumors and relatively small tissue volume with uniform surgical technique might explain why only a trend towards an association was observed between higher excised tissue volume and worse cosmetic outcome.

This study is a small retrospective study and the different follow-up periods of the patients could be a limitation. The small sample size of this study might be the reason for failure to identify a correlation between several surgical and other 
factors and the cosmetic results. Only a slight correlation was observed between patient's self-assessment and the overall result of BCCT.core $(\mathrm{K}=0.12)$ and no significant correlation was observed between patient's self-assessment and the seven quantitative values of breast asymmetry (Table 2). Nonetheless, some of the asymmetry scores showed a negative relationship with the patients' assessment. As demonstrated by these results, subjective assessment of cosmetic outcome may not necessarily agree with the objective scores, therefore cosmetic assessment may need to be compensated and validated for both the patient-reported and objective tool in order to achieve more consistent and significant results. The patient-reported cosmetic outcome was not assessed using a validated written questionnaire, but by a conversation with researchers not involved in treatment of patients. This may cause the subjective outcome to show slight correlation with the result of BCCT.core and the subjective assessment should be compensated in future trials.

Because patients were enrolled during their follow up after completion of treatment, we did not have pre-treatment photographs that could be used for comparison. We are currently conducting a prospective study using BCCT.core software for evaluation of cosmesis before and after radiotherapy with a defined follow-up period. These prospective data will provide more reliable information on factors affecting cosmetic outcome assessed by objective tools of the BCCT. core software.

\section{Conclusion}

Our results show that objective measurement of cosmetic outcome of BCT using the BCCT.core software is feasible, and further suggest that the cosmetic outcome of BCT may be affected by the maximum radiation dose within the breast.

\section{Electronic Supplementary Material}

Supplementary materials are available at Cancer Research and Treatment website (http://www.e-crt.org).

\section{Conflicts of Interest}

Conflict of interest relevant to this article was not reported.

\section{Acknowledgments}

This work was supported by grant (No. 11-2011-014) from the SNUBH Research Fund and the grant (No. 0820010) for Cancer Control Program from Korean Ministry of Health \& Welfare to In Ah Kim.

\section{References}

1. van Dongen JA, Bartelink H, Fentiman IS, Lerut T, Mignolet F, Olthuis $G$, et al. Randomized clinical trial to assess the value of breast-conserving therapy in stage I and II breast cancer, EORTC 10801 trial. J Natl Cancer Inst Monogr. 1992;(11):15-8.

2. Harris JR, Levene MB, Svensson G, Hellman S. Analysis of cosmetic results following primary radiation therapy for stages I and II carcinoma of the breast. Int J Radiat Oncol Biol Phys. 1979;5:257-61.

3. Cardoso MJ, Santos AC, Cardoso J, Barros H, Cardoso De Oliveira M. Choosing observers for evaluation of aesthetic results in breast cancer conservative treatment. Int J Radiat Oncol Biol Phys. 2005;61:879-81.

4. Pezner RD, Patterson MP, Hill LR, Vora N, Desai KR, Archambeau JO, et al. Breast retraction assessment: an objective evaluation of cosmetic results of patients treated conservatively for breast cancer. Int J Radiat Oncol Biol Phys. 1985;11:575-8.
5. Vrieling C, Collette L, Bartelink E, Borger JH, Brenninkmeyer SJ, Horiot JC, et al. Validation of the methods of cosmetic assessment after breast-conserving therapy in the EORTC "boost versus no boost" trial. EORTC Radiotherapy and Breast Cancer Cooperative Groups. European Organization for Research and Treatment of Cancer. Int J Radiat Oncol Biol Phys. 1999;45:667-76.

6. Heil J, Carolus A, Dahlkamp J, Golatta M, Domschke C, Schuetz F, et al. Objective assessment of aesthetic outcome after breast conserving therapy: subjective third party panel rating and objective BCCT.core software evaluation. Breast. 2012;21:61-5.

7. Preuss J, Lester L, Saunders C. BCCT.core: can a computer program be used for the assessment of aesthetic outcome after breast reconstructive surgery? Breast. 2012;21:597-600.

8. Cardoso JS, Cardoso MJ. Towards an intelligent medical 
system for the aesthetic evaluation of breast cancer conservative treatment. Artif Intell Med. 2007;40:115-26.

9. Cardoso MJ, Cardoso J, Amaral N, Azevedo I, Barreau L, Bernardo $\mathrm{M}$, et al. Turning subjective into objective: the BCCT.core software for evaluation of cosmetic results in breast cancer conservative treatment. Breast. 2007;16:456-61.

10. Heil J, Dahlkamp J, Golatta M, Rom J, Domschke C, Rauch G, et al. Aesthetics in breast conserving therapy: do objectively measured results match patients' evaluations? Ann Surg Oncol. 2011;18:134-8.

11. Halperin EC, Wazer DE, Perez CA, Brady LW. Perez and Brady's principles and practice of radiation oncology. 6th ed. Philadelphia, PA: Lippincott Williams and Wilkins; 2012. p. 1808.

12. Hepel JT, Tokita M, MacAusland SG, Evans SB, Hiatt J, Price $\mathrm{L}$, et al. Toxicity of 3D-CRT for accelerated partial breast irradiation. Int J Radiat Oncol Biol Phys. 2008;72:S5.

13. Neal AJ, Torr M, Helyer S, Yarnold JR. Correlation of breast dose heterogeneity with breast size using 3D CT planning and dose-volume histograms. Radiother Oncol. 1995;34:210-8.

14. Moody AM, Mayles WP, Bliss JM, A'Hern RP, Owen JR, Regan $\mathrm{J}$, et al. The influence of breast size on late radiation effects and association with radiotherapy dose inhomogeneity. Radiother Oncol. 1994;33:106-12.

15. Harsolia A, Kestin L, Grills I, Wallace M, Jolly S, Jones C, et al. Intensity-modulated radiotherapy results in significant decrease in clinical toxicities compared with conventional wedge-based breast radiotherapy. Int J Radiat Oncol Biol Phys. 2007;68:1375-80.

16. Donovan E, Bleakley N, Denholm E, Evans P, Gothard L, Hanson J, et al. Randomised trial of standard 2D radiotherapy (RT) versus intensity modulated radiotherapy (IMRT) in patients prescribed breast radiotherapy. Radiother Oncol. 2007;82:
254-64.

17. Vrieling C, Collette L, Fourquet A, Hoogenraad WJ, Horiot JC, Jager JJ, et al. The influence of the boost in breast-conserving therapy on cosmetic outcome in the EORTC "boost versus no boost" trial. EORTC Radiotherapy and Breast Cancer Cooperative Groups. European Organization for Research and Treatment of Cancer. Int J Radiat Oncol Biol Phys. 1999;45:677-85.

18. Romestaing P, Lehingue $Y$, Carrie C, Coquard R, Montbarbon $\mathrm{X}$, Ardiet JM, et al. Role of a 10-Gy boost in the conservative treatment of early breast cancer: results of a randomized clinical trial in Lyon, France. J Clin Oncol. 1997;15:963-8.

19. Taylor ME, Perez CA, Halverson KJ, Kuske RR, Philpott GW, Garcia DM, et al. Factors influencing cosmetic results after conservation therapy for breast cancer. Int J Radiat Oncol Biol Phys. 1995;31:753-64.

20. Hau E, Browne LH, Khanna S, Cail S, Cert G, Chin Y, et al. Radiotherapy breast boost with reduced whole-breast dose is associated with improved cosmesis: the results of a comprehensive assessment from the St. George and Wollongong randomized breast boost trial. Int J Radiat Oncol Biol Phys. 2012;82:682-9.

21. Whelan TJ, Pignol JP, Levine MN, Julian JA, MacKenzie R, Parpia S, et al. Long-term results of hypofractionated radiation therapy for breast cancer. N Engl J Med. 2010;362:513-20.

22. Kim B, Shin SS, Kim SD, Noh DY, Ha SW. Cosmetic results of conservative treatment for early breast cancer. J Korean Soc Ther Radiol Oncol. 2011;19:21-6.

23. Van Limbergen E, Rijnders A, van der Schueren E, Lerut T, Christiaens R. Cosmetic evaluation of breast conserving treatment for mammary cancer. 2. A quantitative analysis of the influence of radiation dose, fractionation schedules and surgical treatment techniques on cosmetic results. Radiother Oncol. 1989;16:253-67. 